\title{
Effects of Sonication Processing on the Behavior of the Synthesis Human Serum Albumin-SPIONs Loaded PLGA Nanoparticles
}

\author{
S. Vidawati ${ }^{1}$, S. Barbosa ${ }^{2}$, P. Taboada ${ }^{2}$, V. Mosquera ${ }^{2}$ \\ ${ }^{1}$ Faculty of Post Graduate, National Institute of Science and Technology, Jakarta, Indonesia \\ ${ }^{2}$ Grupo de Física de Coloides y Polímeros, Departamento de Física de la Materia Condensada, Universidad de Santiago de \\ Compostela, Santiago de Compostela, Spain \\ Email: pablo.taboada@usc.es
}

How to cite this paper: Vidawati, S., Barbosa, S., Taboada, P. and Mosquera, V. (2019) Effects of Sonication Processing on the Behavior of the Synthesis Human Serum Albumin-SPIONs Loaded PLGA Nano- particles. Advances in Biological Chemistry, 9, 179-188.

https://doi.org/10.4236/abc.2019.96014

Received: October 17, 2019

Accepted: November 26, 2019

Published: November 29, 2019

Copyright $\odot 2019$ by author(s) and Scientific Research Publishing Inc. This work is licensed under the Creative Commons Attribution International License (CC BY 4.0).

http://creativecommons.org/licenses/by/4.0/

\begin{abstract}
This paper reports the most prominent contributions in the field of biodegradable polymeric nanoparticles from poly (lactic-co-glycolic acid) (PLGA) used as a protein/drug delivery. We use a combination of Human Serum Albumin (HSA)-superparamagnetic iron oxide nanoparticles (SPIONs) loaded PLGA nanoparticles. To obtain protein stabilization, the optimization of each step of synthesis nanoparticle is required. One of the most common problems in encapsulating protein to PLGA nanoparticles is the presence of several challenges as a problem of instability. We explained how the effect of the various sonication processing on the synthesis HSA-SPIONs loaded PLGA nanoparticles would be one of the crucial parameters for stability.
\end{abstract}

\section{Keywords}

Nanoparticles, PLGA, SPIONs, HSA, Sonication

\section{Introduction}

Nanoparticles are dense and spherical structures range from $100 \mathrm{~nm}-200 \mathrm{~nm}$ in size and are made from natural or synthetic polymers. Various medications can be delivered using nanoparticles, such as hydrophilic small drug, hydrophobic small drug, vaccines, and biological macromolecules. Nanoparticles also allow the administration of specific organs or cells or controlled drug delivery.

In connections with the safety of the polymers used for encapsulation, Poly (lactic-co-glycolic acid) (PLGA) is one of the most successfully used biodegradable polymers, because its hydrolysis leads to metabolite monomers, lactic acid 
and glycolic acid. PLGA has been selected to design nanoparticles as the drug delivery systems in variety of biomedical applications, such as vaccination, cancer, inflammation and other diseases. PLGA is approved by the US FDA and European Medicine Agency (EMA) in a variety of drug delivery systems in humans.

The development of nanotechnology is explained in medical sciences, e.g. SPIONs (Superparamagnetic Iron Oxide Nanoparticles). SPIONs appear with significant potential application in Magnetic Resonance Imaging (MRI), drug delivery, magnetic hyperthermia, tissue repair, detoxification of biological fluids, and in cell separation, etc. The development of nanoparticles for the delivery of contrast agents has emerged in recent years because of the possibility of producing multifunctional nanoparticles that can specifically target tumors [1]. PLGA is used to formulate nanoparticles that encapsulate superparamagnetic iron oxide for MRI. This system enhances the imaging effect along with increasing the half-life of nanoparticles in the bloodstream, thereby reducing side effects [2].

This encapsulation of these therapeutic proteins in PLGA nanoparticles has emerged as a promising alternative to overcome all these problems as well as to contribute with certain additional benefits. Combining proteins into the polymer matrix provides protection against enzymatic and hydrolytic degradation in vi$v o$, maintains their integrity and activity, can increase their bioavailability and in some cases can target therapeutic protein to the target area.

Biodegradable nanoparticles production contains stable therapeutic proteins, mostly in terms of technical barriers. The precise assessment of the stability and quantifying of protein encapsulation remains difficult for major tasks and barrier prior to analysis [3] [4] [5] [6]. To enable protein stabilization, the optimization of each step of nanoparticles production is required. One of the most common techniques for encapsulating proteins into PLGA nanoparticles presents several challenges as a matter of instability [7]. Often protein instability is closely related to the presence of water or interfaces during particle preparation and some new techniques. Proteins from therapeutic should be studied on a case-by-case basis, so as to bring to the stage of future processing and stress factors that damage them.

To address this problem, many studies have focused on optimizing the formulation process in order to improve protein stability during the processing of procedures. The purpose of this study is investigated the effect of sonication processing on the behavior of the encapsulated PLGA nanoparticles for protein/drug delivery. For this study, we used HSA as a protein model. We use encapsuled PLGA loaded combinations of SPIONs and HSA. These nanoparticles are characterized for their physicochemical properties.

The importance of mixing kinetics solution containing hydrophobic and non-solvent compounds is highlighted. They require accurate processing parameters of possible sonication of fabrication for applications, nanoparticulate delivery systems. Sonication is used in a variety of physical, chemical and biological processes. Sonication is a highly effective processing method for generation and the application of nanosize, homogenizing, emulsifying, and dispersing for physical 
processes. Sonication with high-powered pulses is used to increase the dispersion of nanoparticles in the preparation of nanofluids. High-intensity sonication is used for the processing of liquids such as mixing, emulsifying, dispersing and de-agglomeration, or milling. When liquids are sonicated with high intensity, the sound waves that spread to the liquid media produce high-pressure (compression) and low-pressure (rarefaction) cycles, with rates depending on the frequency. Variations of the sonication intensity probe are studied to determine its effect on the characteristics of nanoparticles, such as average agglomerate size, polydispersity of the solution, and surface charge. The processing conditions have an important effect on the morphology, particle size and the formation of a stable nanoparticles phase.

\section{Materials and Methods}

\subsection{Materials}

PLGA of 38 - $54 \mathrm{kDa}$ with 50:50 lactide-glycolide ratio, Pluronic $\mathrm{F}_{127}, \mathrm{FeCl}_{2}$, $\mathrm{FeCl}_{3}$, and Human Serum Albumin (HSA), obtained from Sigma-Aldrich (St. Louis, MO, USA). Oleic acid with purty $90 \%$ obtained from Alfa Aesar (Karlshrue, Germany). All other chemicals and solvents obtained from Sigma-Aldrich. Pure water of Milli-Q quality is used in all preparations.

\subsection{Synthesis of SPIONs}

Oleic acid-stabilized $\mathrm{Fe}_{3} \mathrm{O}_{4}$ SPIONs are synthesized with the method co-precipitation. In summary, an aqueous solutions of $0.1 \mathrm{M}$ of $\mathrm{FeCl}_{3}(30 \mathrm{~mL})$ and $\mathrm{FeCl}_{2}(15 \mathrm{~mL})$ prepared with $\mathrm{N}_{2}$ purged-water were mixed; then, $3 \mathrm{~mL}$ of 5 $\mathrm{M}$ solution of ammonia was added in small aliquots of $0.6 \mathrm{~mL}$ while stirring. A black precipitate is made indicating the formation of SPIONs. After $20 \mathrm{~min}$ of stirring under $\mathrm{N}_{2}$ atmosphere, $56.4 \mathrm{mg}$ of oleic acid was added to the SPIONs and the temperature increased to $80^{\circ} \mathrm{C}$ and kept for $30 \mathrm{~min}$ while stirring to evaporate the ammonia. The magnetic nanoparticles were washed twice by centrifugation at $9000 \mathrm{rpm}$ for $20 \mathrm{~min}$ and the precipitate was lyophilized and stored at $4^{\circ} \mathrm{C}$.

\subsection{Synthesis of HSA-SPIONs-PLGA Nanoparticles}

Preparation of the polymer is encapsulated PLGA nanoparticles containing a combination of SPIONs and HSA prepared by using the multiple emulsion solvent evaporation methods. In typical preparation, PLGA $(25 \mathrm{mg})$ dissolved in a sealed vial containing Dichloromethane $(1 \mathrm{ml})$, HSA dissolved in pure water $(100 \mu \mathrm{L})$ by ultrasonic $10 \mathrm{~min}$, and SPIONs dispersed in Dichloromethane by sonication with a probe type sonicator $(20 \mathrm{kHz}$, Bandelin Sonopuls, Bandelin $\mathrm{GmbH}$, Berlin, Germany) on some parameters of time and power in an ice bath. The combinations of time variation parameters and power of sonication with accurately is crucial to produce a very high quality of polymeric PLGA nanoparticles with containing combinations of SPIONs and HSA. In this study showed 
that the difference in the power and timing of sonication parameters are very small could yield significantly very different result from each synthesis of HSA-SPIONs-PLGA nanoparticles.

Then, this organic solution added a wise drop with a syringe pump (0.166 $\mathrm{mL} / \mathrm{min}$ ) to an aqueous solution $\left(50 \mathrm{~mL}\right.$ ) containing Pluronic $\mathrm{F}_{127}$ (typically 1 $\mathrm{wt} \%$ if not otherwise stated) while stirring at $10^{\circ} \mathrm{C}$. After sonication with power $100 \mathrm{~W}$ for 15 minutes from this experiment to homogenize the resulting dispersion, the organic solvent completely evaporated under mechanical stirring overnight, the dispersion subsequently centrifuged twice at $9000 \mathrm{rpm}$ for $20 \mathrm{~min}$ and $20^{\circ} \mathrm{C}$. Subsequently, the supernatant was removed and the final precipitates are stored in the freezer.

\subsection{Characterization of Nanoparticles}

In this study, all of nanoparticles were characterized using TEM image, Zeta Potential, and UV-Vis spectroscopy measurements.

The Transmission electron microscopy (TEM) is used for described particle size and morphology of nanoparticles. Samples are prepared for analysis by evaporation a nanoparticles dispersion on a carbon-coated cooper grid without staining (TEM). TEM images of nanoparticles are obtained by a Philips CM-12 (Philips, Netherlands) microscope operating at $120 \mathrm{kV}$. HR-TEM images and selected area electron diffraction (SAED) patterns obtained with the transmission electron microscope (Carl-Zeiss Libra 200 FE-EFTEM, Germany) operating at $200 \mathrm{kV}$.

UV-Vis measurements are used to describe Human Serum Albumin (HSA) released. UV-Vis spectroscopy measurements were performed in a CARY 100 Bio UV-Visible (Agilent Technologies, Santa Clara, USA) spectrophotometer.

The zeta potentials of nanoparticles are obtained by triplicate with a Zetasizer Nano ZS (Malvern, UK), using disposable folded capillary cells. Each experiment is repeated at least three times.

\section{Result and Discussion}

\subsection{SPIONs}

In this study, oleic acid-stabilized SPIONs were obtained with a co-precipitation method [3]. Without the coating, SPIONs tend to be aggregated, they are also hydrophobic and, when injected into the bloodstream, are coated by plasma proteins (called opsonization). The hydrophilic coating may prevent or significantly reduse opsonization, and through electrostatic interactions or steric hindrance, it decreases the aggregation of the SPIONs.

TEM image of the size and morphology of SPIONs is shown in Figure 1. Transmission electron microscopy (TEM) measurement shows the spherical morphology of hematite nanoparticles and narrow size distribution. TEM characterization of SPIONs was shown sized particle with a mean diameter of SPIONs around $5-20 \mathrm{~nm}$. The SPIONs (Figure 1) are seemingly uniform and stable in 


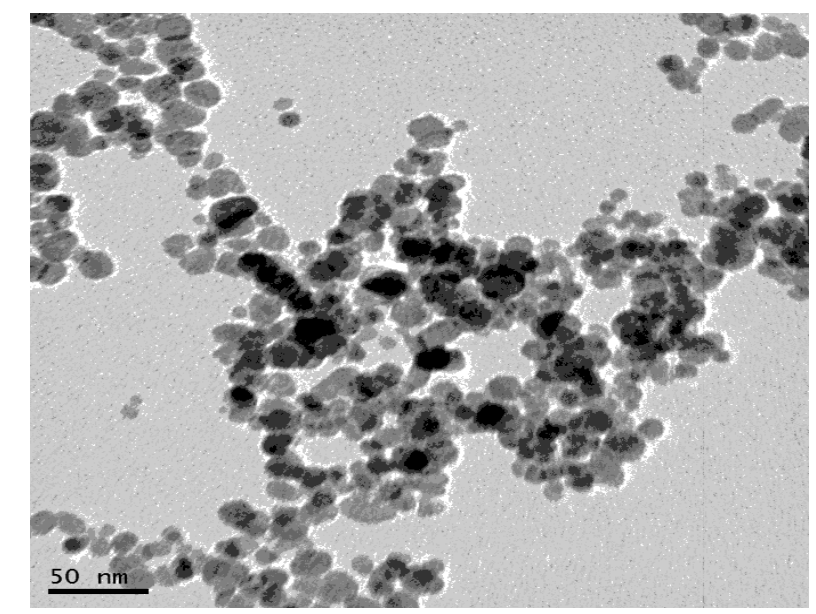

Figure 1. TEM image of superparamagnetic iron oxide nanoparticles.

the size and shape. The magnetic microspheres of SPIONs (with diameter size of $5-20 \mathrm{~nm}$ ) in biocompatible, non-toxic (FDA approved) and biodegradable polymeric microspheres, such as polymeric PLGA nanoparticles are recognized as desirable promising for application in spintronics and biomedicine.

The Zeta potential of SPIONs in this study around $+45 \mathrm{mV}$, the zeta potential representing a surface charge of the particles in a colloidal suspension, is one of the most important factors defining their stability, a tendency to aggregate (thus defines them effective size), as well as their ability to bind serum proteins. In spite of the fact that most of them are charged negatively, the more positive the charge of a SPION is, the stronger its ability to bind serum proteins [8].

\subsection{HSA-SPIONs Loaded PLGA Nanoparticles}

Many studies have been studied further on the combination of PLGA nanoparticles with SPIONs and protein or vaccines or amongst others [3] [9]. Emulsification by sonication a preparation method to the drug-loaded systems of biodegradable PLGA nano-carriers contains SPIONs and HSA. Parameter preparation for the formation of PLGA nanoparticles for protein delivery

In this study, we were informed about the effect of sonications on the behavior of synthesis biodegradable PLGA nanoparticles for protein delivery using a combination of SPIONs and HSA. We used various of sonication parameters on the each synthesis encapsulated processing.

Sonication with a type of sonicator probe $(20 \mathrm{kHz}$, Bandelin Sonopuls, Bandelin $\mathrm{GmbH}$, Berlin, Germany) is used. Sonication largely affects the reaction rate, yields, and is influenced by polymeric PLGA nanoparticles for protein delivery. The results of this passage are derived that the parameters of the sonication intensity processing become an important parameter on the synthesis biodegradable polymeric HSA-SPIONs loaded PLGA nanoparticles. This study suggests that a variety of power and timing parameters of sonication can produce significant result (particle size and stability) from synthesis of polymeric PLGA nanoparticles containing SPIONs and HSA. The combinations of time variation pa- 
rameters and the power sonication accurately are crucial to produce the polymeric HSA-SPIONs loaded PLGA nanoparticles.

The ultrasonic emulsification has been studied for decades and has recently garnered increased interest [10] [11]. The study compares the ultrasonic emulsification with the dispersing rotors [11] [12] finding ultrasound to be competitive or even superior in terms of droplet size and energetic efficiency. Oil-in-water emulsions, the system is also found useful for biodegradable nanoparticles preparations using the solvent extraction/evaporation method. The sonication power is controlled by the transfer oscillation amplitude. To measure the power consumed for emulsification, the power intake of a high frequency generator was recorded using a standard household power monitor. For $100 \%, 80 \%$ and $60 \%$ of the maximum amplitude, the power intake is of $32 \mathrm{~W}, 25 \mathrm{~W}$ and $17 \mathrm{~W}$, respectively. The actual power assessment transferred to the emulsion is usually done by measuring the heat taken by the emulsion, which for current ultrasonic flow-through cell will be difficult to do with reasonable accuracy. However, it makes sense to assume that the power consumption by the generator should be comparable to that delivered to the emulsion [13] [14]. PLGA nanoparticles, forming emulsion in ultrasonic flow-cell via rapid, oil-in-water emulsions, the particle sizes is increased with less sonication power, although the difference is less pronounced than observed for the oil emulsions. By increasing the concentration of the polymer solution, and hence its viscosity, larger particles are produced. Obviously, viscosity is not the only physicochemical. Set the emulsification parameter, as it has been noted for the oil-in-water emulsion. Factors such as the tension of the interface and surfactants conformity are equally important, especially with regard to droplet coalescence.

The size and morphology of HSA-SPIONs loaded PLGA nanoparticles of this experiment is characterized by TEM. TEM image are used to obtain important information about the main size and morphology of nanoparticles. TEM is an important technique whose unique ability to probing the internal structure of individual nanoparticles.

The results TEM images of HSA-SPIONs loaded PLGA nanoparticles are displayed interesting phenomenon information in the synthesis of HSA-SPIONs loaded PLGA nanoparticles processing with the sonication variation parameters. Figure 2 to number 5 has provided information about TEM image $0.05 \mathrm{~g} / \mathrm{ml}$ HSA-SPIONs loaded PLGA nanoparticles which multple parameters of sonication processing combinations for mixture the PLGA + SPIONs + HSA solutions and in vitro release of HSA from HSA-SPIONs loaded PLGA nanoparticles. In this study, HSA-SPIONs loaded PLGA nanoparticles had a zeta potential between $-4.2 \mathrm{mV}$ until $-10.15 \mathrm{mV}$.

The first variation of sonication in the synthesis polymeric was $0.05 \mathrm{~g} / \mathrm{ml}$ HSA-SPIONs-PLGA nanoparticles shown in Figure 2. PLGA + SPIONs + HSA solutions mix with sonication parameter in the first power $20 \mathrm{~W}$ for 10 minutes, than second power $60 \mathrm{~W}$ for 4 minutes in an ice bath. TEM image in Figure 2 is done that polymeric PLGA nanoparticles appear in the sphere nanocapsule, 


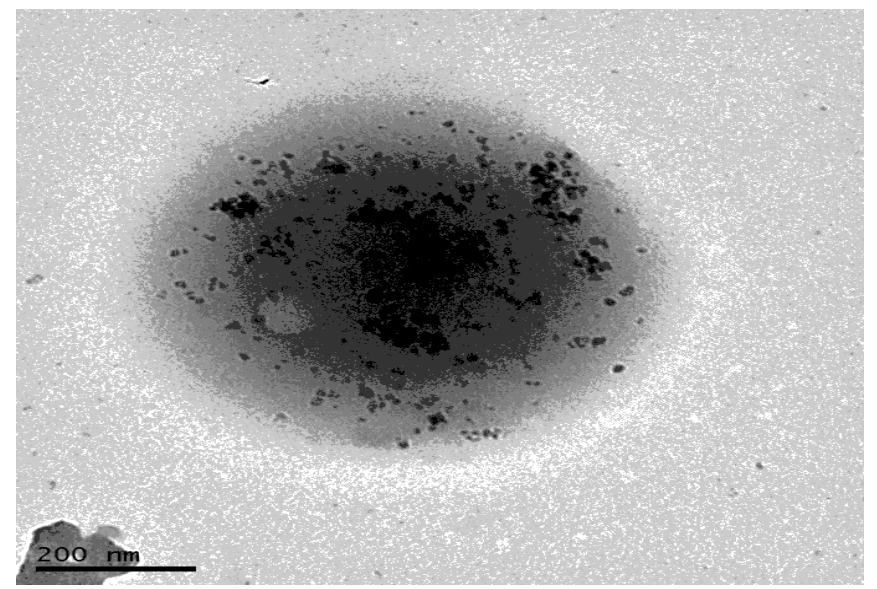

Figure 2. TEM images of HSA-SPIONs loaded PLGA nanoparticles with sonication step in the first power of $20 \mathrm{~W}$ for $10 \mathrm{~min}$, than the second power of $60 \mathrm{~W}$ for $4 \mathrm{~min}$.

but from UV-Vis measurement results are notified that HSA does not contain in the polymeric PLGA nanoparticles.

Another parameter of the sonication variation for PLGA + SPIONs + HSA solutions displayed TEM image Figure 3. PLGA + SPIONs + HSA is a mixture with sonication parameters: the first power of $60 \mathrm{~W}$ for 2 minutes and a second power of $20 \mathrm{~W}$ for 10 minutes in an ice bath. TEM image is performed polymeric $0.05 \mathrm{~g} / \mathrm{ml} \mathrm{HSA-SPIONs-PLGA}$ nanoparticles on this parameter as a donut structure (Figure 3). PLGA + SPIONs + HSA solutions is mix with parameters similar to previous sonication parameters as in Figure 3, but the results are significant different obtained from UV-Vis measurement. Unfortunately, from the UV-Vis spectrometry, it does not contain HSA in the polymeric PLGA nanoparticles.

Figure 4 shows the result of sonication variation using the first power of $60 \mathrm{~W}$ for 1 minute, than the second power of $20 \mathrm{~W}$ for 10 minutes in an ice bath. The TEM morphology image of nanoparticles in Figure 4(a) is displayed spherical. They have a heterogeneous structure. Polymeric HSA-SPIONs loaded PLGA nanoparticles have a donut-shaped structure, and another one has perfect round nanoparticle. UV-Vis measurement results indicate that the HSA in the PLGA nanoparticles only 50 percent. In vitro the release of HSA profile was observed, and all result of this study according to Gopferich et al. [14] about the release of protein. From biodegradable nanoparticles in Figure 4(a), HSA release around $5.5 \%$ to 120 hours (see Figure 4(b)).

Figure 5 performed TEM image of $0.05 \mathrm{~g} / \mathrm{ml}$ HSA-SPIONs loaded PLGA nanoparticles look perfect in the sphere nanocapsule. We used the sonication parameters to mix the PLGA + SPIONs + HSA solutions in the power of $20 \mathrm{~W}$ for 12 minutes in an ice bath (Figure 5(a)). UV-Vis measurement results show that the HSA in the polymeric PLGA nanoparticles is so perfect about 99 percent. The release profile is conducted, HSA release about 15\% to 70 hours (see Figure $5(\mathrm{~b}))$. 


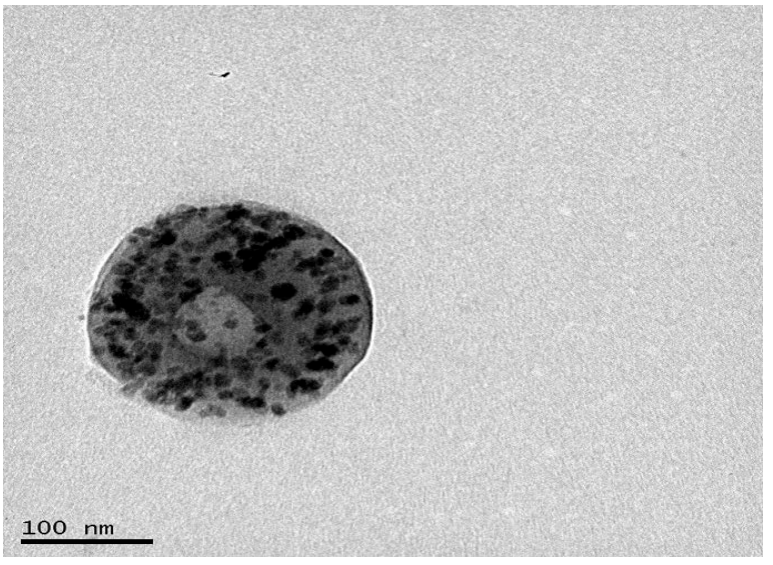

Figure 3. TEM images of HSA-SPIONs loaded PLGA nanoparticles with sonication step in the first power of $60 \mathrm{~W}$ for $2 \mathrm{~min}$, than the second power of $20 \mathrm{~W}$ for $10 \mathrm{~min}$.

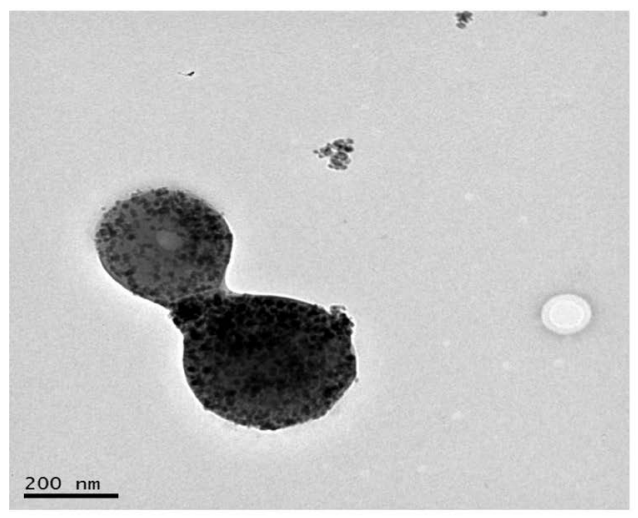

(a)

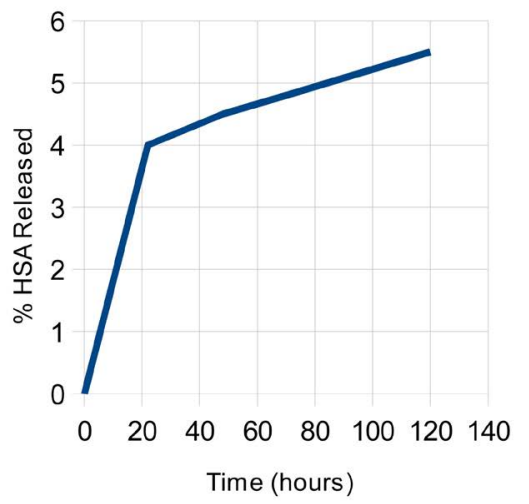

(b)

Figure 4. TEM images of HSA-SPIONs loaded PLGA nanoparticles with sonication step in the first power of $60 \mathrm{~W}$ for $1 \mathrm{~min}$, than the second power of $20 \mathrm{~W}$ for $10 \mathrm{~min}(4 \mathrm{a})$ and in vitro release of HSA from HSA-SPIONs loaded PLGA nanoparticles (4b).

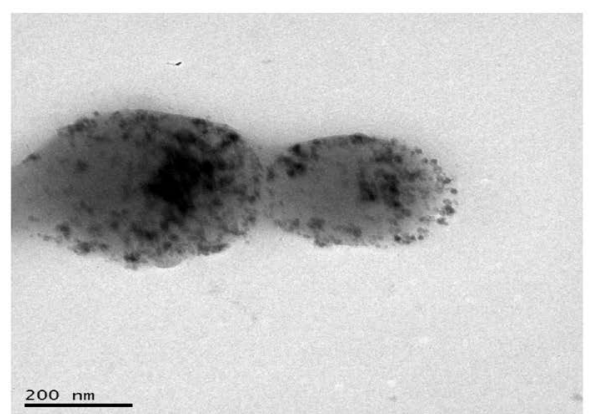

(a)

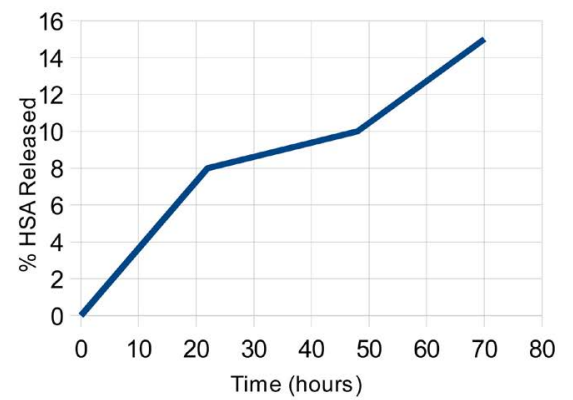

(b)

Figure 5. TEM images of HSA-SPIONs loaded PLGA nanoparticles with sonication step in the power of $20 \mathrm{~W}$ for $12 \mathrm{~min}(5 \mathrm{a})$ and in vitro release of HSA from HSA-SPIONs loaded PLGA nanoparticles (5b).

\section{Conclusion}

Summarizing our data, we argue that the sonication step is to be an important parameter on the synthesis HSA-SPIONs loaded PLGA nanoparticles. The 
tion of sonication power in the processing of synthesis nanoparticles into a crucialparameter obtains excellent results and stability in polymeric HSA-SPIONs loaded PLGA nanoparticles. These results have important implications for their potential applications such as protein/drug delivery.

\section{Acknowledgements}

This study was supported by the Erasmus Mundus II EXPERTS III (SV).

\section{Conflicts of Interest}

The authors declare no conflicts of interest regarding the publication of this paper.

\section{References}

[1] Acharya, S. and Sahoo, S.K. (2011) PLGA Nanoparticles Containing Various Anticancer Agents and Tumour Delivery by EPR Effect. Advanced Drug Delivery Reviews, 63, 170-183. https://doi.org/10.1016/j.addr.2010.10.008

[2] Wang, Y., Ng, Y.G., Chen, Y., Shuter, B., Yi, J., Ding, J., Wang, S. and Feng, S.G. (2008) Formulation of Superparamagnetic Iron Oxides by Nanoparticles of Biodegradable Polymers for Magnetic Resonance Imaging. Advanced Functional Materials, 18, 308-318. https://doi.org/10.1002/adfm.200700456

[3] Vidawati, S., Barbosa, S., Taboada, P., Topete, A., Villar, E. and Mosquera, V. (2018) Study of Human Serum Albumin-SPIONs Loaded PLGA Nanoparticles for Protein Delivery. Advances in Biological Chemistry, 8, 91-100. https://doi.org/10.4236/abc.2018.85008

[4] Wolf, M., Wirth, M., Pittner, F. and Gabor, F. (2003) Stabilisation and Determination of the Biological Activity of L-asparaginase in Poly(D,L-lactide-co-glycolide) $\mathrm{Na}$ nospheres. International Journal of Pharmaceutics, 256, 141-152. https://doi.org/10.1016/S0378-5173(03)00071-1

[5] Park, T.G., Lu, W. and Crotts, G. (1995) Importance of in Vitro Experimental Conditions on Protein Release Kinetics, Stability and Polymer Degradation in Protein Encapsulated Poly(D,L-lactic acid-co-glycolic acid) Microspheres. Journal of Controlled Release, 33, 211-222. https://doi.org/10.1016/0168-3659(94)00084-8

[6] Crotts, G. and Park, T.G. (1998) Protein Delivery from Poly(lactic-co-glycolic acid) Biodegradable Microspheres: Release Kinetics and Stability Issues. Journal of Microencapsulation, 15, 699-713. https://doi.org/10.3109/02652049809008253

[7] Van de Weert, M., Hennink, W.E. and Jiskoot, W. (2000) Protein Instability in Poly(Lactic-Coglycolic Acid) Microparticles. Pharmaceutical Research, 17, 1159-1167. https://doi.org/10.1023/A:1026498209874

[8] Bravo-Osuna, I., Ponchel, G. and Vauthier, C. (2007) Tuning of Shell and Core Characteristics of Chitosan-Decorated Acrylic Nanoparticles. European Journal of Pharmaceutical Sciences, 30, 143-154. https://doi.org/10.1016/j.ejps.2006.10.007

[9] Topete, A., Melgar, D., Alatorre-Meda, M., Iglesias, P., Argibay, B., Vidawati, S., Barbosa, S., Costoya, J.A., Taboada, P. and Mosquera, V. (2014) NIR Light Active Hybrid Nanoparticles for Combined Imaging and Bimodal Therapy of Cancerous Cells. Journal of Materials Chemistry B, 2, 6967-6977. https://doi.org/10.1039/C4TB01273A

[10] Medina, J., Salvado, A. and del Pozo, A. (2001) Use of Ultrasound to Prepare Lipid 
Emulsions of Lorazepam for Intravenous Injection. International Journal of Pharmaceutics, 216, 1-8. https://doi.org/10.1016/S0378-5173(00)00664-5

[11] Maa, Y.F. and Hsu, C.C. (2001) Performance of Sonication and Microfluidization for Liquid-Liquid Emulsification. Pharmaceutical Development and Technology, 4, 233-240. https://doi.org/10.1081/PDT-100101357

[12] Abisma1, B., Canselier, J.P., Wilhelm, A.M., Delmas, H. and Gourdon, C. (1999) Emulsification by Ultrasound: Drop Size Distribution and Stability. Ultrasonics Sonochemistry, 6, 75-83. https://doi.org/10.1016/S1350-4177(98)00027-3

[13] Ratoarinoro, F., Contamine, A.M., Wilhelm, J., et al. (1995) Power Measurement in Sonochemistry. Ultrasonics Sonochemistry, 2, S43-S47. https://doi.org/10.1016/1350-4177(94)00010-P

[14] Loning, J.M., Horst, C. and Hoffmann, U. (2002) Investigations on the Energy Conversion in Sonochemical Processes. Ultrasonics Sonochemistry, 9, 169-179. https://doi.org/10.1016/S1350-4177(01)00113-4 